Digital Press Social Sciences and Humanities

La question cruciale pour son propre pays : analyse du discours critiques sur le roman Le Mouchoir par Mohamed Kacimi

Ida Bagus Putra Ananta Sidhiswara

Proceeding of Conférence internationale sur le français 2018

Joesana Tjahjani, Merry Andriani, Sajarwa, Wening Udasmoro (eds) 


\title{
La question cruciale pour son propre pays : analyse du discours critiques sur le roman Le Mouchoir par Mohamed Kacimi
}

\author{
Ida Bagus Putra Ananta Sidhiswara \\ Universitas Gadjah Mada, Yogyakarta, Indonesia \\ *e-mail : gustuwino@gmail.com
}

\begin{abstract}
Résumé
Chaque phrase choisie contient une motivation et idéologie de l'écrivain. Et celle-ci n'est pas évidente, elle n'est pas explicite. On peut la trouver dans la structure de l'histoire, les textes linguistiques qui crée un Discours dont les idées construisent. Kacimi à l'âge de 25 ans, a quitté son pays et est parti pour la France. Il a publié son premier roman qui s'appelle Le Mouchoir dans lequel il tente de critiquer la société algérienne, son gouvernement et ses concitoyens de manière caricaturale et satirique. Cette recherche analyse la critique évoquée dans le roman. Le but de cette recherche est de révéler comment l'écrivain critique son propre jeune pays, après l'avoir quitté, et également les formes de critiques qui sont cachées dans les symboles au regard du narrateur. Cette recherche utilise la théorie d'analyse du discours critiques de Fairclough qui révèle la perspective de l'auteur dans les mots, phrases, ou paragraphes. Cette recherche révèle les critiques pour un pays dont l'écrivain parle dans le roman aux yeux du narrateur, ainsi que les comportements indifférents et insolents des citoyens, la dictature du peuple, l'état totalitaire qui se trouvent dans l'histoire. Le résultat a prouvé que ce roman est une critique d'un pays totalitaire sous la forme d'un récit irréel. Tout cela se retrouve dans le titre, les étages, les noms des personnages, et le lieu.
\end{abstract}

\section{Mots-clés}

critique, discours, Mouchoir, Kacimi, Algérie

\begin{abstract}
Every sentence selected contain a desire and an ideology of the author. This one is not obvious; it is not explicitly mentioned. We can find it in the structure of the story, words and phrases used to define the object of the critique. Leaving his country at the age of 25, Kacimi went to France. He published his first novel entitled Le Mouchoir where he tries to criticize the Algerian society, the government and his compatriots in a caricatured way and using satire. This research analyses the critics mentioned in the novel. The aim of this research is to reveal how the author criticizes his proper country after he leaved it and the hidden forms of critics under the symbols from narrator's regard. This research utilizes the theory of Critical Discourse Analysis from Fairclough that reveals the perspective of the author in words, sentences, and paragraphs. This research revealed the critics for a country that the author talks in the novel by the regards of narrator, also the indifferent and insolents behaviors of the citizens, the dictator of the people, totalitarian country that we can see in the story. The result proved that this novel is a critic to a totalitarian country in the form of fictive story. All of these can be found in the title, stages, characters, and the space.
\end{abstract}

\section{Keywords}

critic, discourse, Mouchoir, Kacimi, Algeria 


\section{Introduction}

Chaque pays à sa propre histoire qui a pour origine une civilisation propre qui évolue et se produit tout au long de son existence. Son type de gouvernement, sa culture, sa coutume, sa géographie, et ses citoyens sont les éléments importants qui participent au développement d'un pays. Et ceux-ci peuvent être transformés surtout si ces substances rencontrent d'autres substances ayant différentes caractéristiques qui se mélangent, et font naître un système nouveau.

Les œuvres francophones qui viennent des pays ex-colonisation française ou départements d'outremer sont toujours intéressants à analyser. L'auteur a un point de vue différent et se penche sur les problèmes sociaux qui existent au sein de sa société d'origine ou sur sa relation avec son ancien camarade, la France.

Surtout, cela est encore plus vrai quand des expatriés décident de vivre en France et rédige un récit critique sur son pays d'origine d'une manière caricaturale et satirique.

Nous ne renierons pas qu'il y a toujours des raisons qui pousse à quitter sa terre natale, que des problèmes surgissent et sont en opposition avec ses pensées ou qui appellent même à la violence. Il ne supporte plus toutes les structures dans son pays.

Un écrivain Algérien Mohamed Kacimi El-Hassani a décidé de quitter son pays, l'Algérie, en 1982 pour vivre à Paris. Avant le départ pour le pays des penseurs, la France, il avait déjà fait ses études en littérature française à l'École Normale Supérieure de Kouba. Il publia son premier roman en 1987 en France après avoir eu plusieurs rejets de publication dans son pays natal (CRIS (Centre de Ressources Internationales de la Scène), s .d.)

Le Mouchoir raconte une histoire d'un fonctionnaire (le narrateur anonyme) qui travaille pour un Parti Socialiste en Algérie. Il fait tout pour garder pour garder l'honneur du Parti. C'est lui seul qui témoigne d'une réalité dégoûtante dans les toilettes du bâtiment du Parti. Une tache de sperme qui risquerait de tâcher la dignité du Parti et que ce fonctionnaire la garde en utilisant un mouchoir. Ce mouchoir le rend obsessif jusqu'à ce qu'il oublie de garde son honneur.

Les aspects intéressants sont la vie bureaucrate, la misère incessante, la société algérienne décrite par le narrateur anonyme qui est également le personnage principal. Et presque tous les personnages sont des anonymes cachés par le nom de leurs postes ou leur relation avec le narrateur comme le commissaire, la secrétaire, sa femme, son beau-père. Il n'y a qu'un personnage actif nommé Abdou, ainsi que d'autres qui sont passifs nommés Mahfoud et Montalier.

\subsection{La problématique}

Le narrateur raconte sa vie quotidienne au travers de sa vie professionnelle, familiale, et son côté obscur. Mais sachant qu'un roman n'est qu'une histoire irréelle, il y a quelques ressemblances sur ce qu'il raconte avec la réalité (la société algérienne et la biographie de l'écrivain). Il y a une critique cachée qui se cache dans sa manière de raconter son histoire. Basée sur cette problématique, on peut se poser les questions suivantes :

1. Comment l'auteur critique-t-il son propre pays?

2. Quels sont les symboles critiques représentés dans le roman?

\subsection{L'objectif}

Cette recherche a pour but de découvrir et dévoiler les idées cachées de l'auteur par le biais de la narration, et sur son propre pays à travers les discours dans le roman.

\subsection{La Méthode}

Tout d'abord, le déroulement de cette recherche commence par la première lecture pour connaitre le fil conducteur de l'histoire. Puis, cela continue avec la deuxième lecture en observant et interprétant les discours portés par les mots, les phrases, et les paragraphes dans le but de trouver les données critiques et les symboles critiques dans les discours. Ensuite, les données récupérées sont recueillies, en utilisant le point de vu d'une analyse de discours critiques. Celles-ci sont analysés durant la relecture, puis en donnant une interprétation. 


\subsection{La Théorie}

L'Analyse du discours critique essaie de percer les pratiques sociales à travers de l'emploi linguistique de l'écrivain. Un discours a tendance à constituer la société et à être constitué par la société elle-même. Cette pratique du Discours crée une représentation du monde selon les sujets et ses relations sociales pour imposer un pouvoir ou l'hégémonie (Fairclough, 1995). L'écrivain, en construisant un Discours, utilise ses regards, l'expérience de ses relations sociales et son idéologie sur le monde et il essaie de le changer avec ce Discours.

\section{Analyse}

\subsection{Les Rapports avec l'Algérie}

Malgré l'absence de la date et du pays, on peut considérer que le lieu et le temps qui sont décrits dans ce roman se passent après l'indépendance de l'Algérie ou bien en Algérie. Cela se voit lors d'une rencontre du narrateur avec Abdou où celui-ci raconte son combat contre les Français mais à la fin le narrateur comprend que cela n'est qu'un mensonge, Abdou n'a jamais tiré avec un pistolet/ une arme à feu. Cela se voit aussi quand le narrateur part avec son ami Mahfoud à Oran (Kacimi, 1987, p. 39), qui se trouve évidemment en Algérie.

Derrière l'histoire de Le Mouchoir contient des intrigues qui révèlent que le fil conducteur n'est pas vraiment sur l'histoire d'un mouchoir mais sur les passages que le narrateur décrit où sur des faits symboliques et des critiques vers son propre pays. Les choses comme un mouchoir, le fait sexuel, les personnages, les lieux contribuent aux données symboliques que l'on peut trouver dans les mots, les phrases et les paragraphes.

L'histoire commune entre ce roman et la réalité se trouve sur l'histoire d'un fonctionnaire qui travaille dans un Parti Socialiste avec l'histoire d'Algérie où le Parti FLN (Front de Libération Nationale) qui est aussi un parti socialiste, un moment après la guerre d'indépendance, a réussi gagner le public et s'imposer son pouvoir en Algérie (Blanchard \& Thénault, 2011, p. 3). Par la suite il déclara une identité arabe dans ce pays africain et commença la politique d'arabisation (Zenati, 2004, p. 138). En revanche, il y a des algériens qui ne veulent pas être considéré en tant qu'Arabe. Ce sont les kabyles (Kaltbrunner, 1871, p. 5).

\subsubsection{Une critique de la culture ridicule d'une façon caricaturale}

Premièrement, ce roman consiste en 6 chapitres nommés en jours de la semaine, commençant par samedi, dimanche, lundi, mardi, mercredi, et jeudi pour donner l'effet d'une société distincte. Il manque le vendredi car le narrateur prétend que c'est un jour férié. Donc Il ne travaille ni ne raconte son histoire dans ce jour.

"Mais depuis que l'Etat a décidé, par souci d'authenticité, de réagencer l'ordre des jours en décrétant le vendredi jour férié... » (Kacimi, 1987, p. 5)

«Victime des habitudes anciennes, je n'ai pu me faire à ce changement» (Kacimi, 1987, p. 5)

Cette citation indique la décision de l'État en déplaçant le jour férié du dimanche au vendredi. Puisque l'histoire se passe après l'indépendance ou l'après-colonisation française, on cherchait à s'identifier autrement que par les caractéristiques du gouvernement colon. Cela représente une critique de l'État, en raison de l'authenticité de son pays qui éradique tout ce qui est lié à la colonisation française et qui semble étranger aux regards Algériens.

"Nul besoin de se laver le visage, il suffit de traîner les pieds dans l'eau des ablutions par qui s'inonde la salle de bains! Le traitement garantit une parfaite lucidité. Mais cette lancée s'estompe dès qu'il s'agit d'atteindre la cuisine. Prendre son café suppose d'abord de passer sur le corps des autres membres de la famille endormis dans le couloir » (Kacimi, 1987, p. 6) «L'apothéose c'est la cigarette. Pour en jouir il faut presser la sortie du père » (Kacimi, 1987, p. 6)

Il existe dans le roman une coutume ridicule dont l'auteur essaie de tourner en critique de la société ayant soif de respect. «Nul besoin de se laver le visage, il suffit de traîner les pieds dans l'eau des ablutions 
par qui s'inonde la salle de bains! » on peut trouver une critique dans cette phrase. Le mot « Le visage » fait de la partie du corps la plus importante qui représente l'image (l'identité) d'une personne. Souvent, les humains se jugent les uns et les autres lors de leur première rencontre, donc l'image a un rôle important dès la première interaction.

En revanche, l'image d'après le narrateur dans cette phrase ne sert à rien : « Il suffit de traîner les pieds dans l'eau des ablutions par qui s'inonde la salle de bains ! Le traitement garantit une parfaite lucidité. » L'eau des ablutions que les hommes font, chez les musulmans, est un symbole de prière. Au regard du narrateur, en se lavant avec l'eau des ablutions est plus important que l'image que les hommes doivent garder. Il prend le plus haut niveau et le remplace par ce qui est moins important. Le caractère sacré de cette action devient meilleur que les autres comme ce qui est décrit dans la phrase « le traitement garantit une parfaite lucidité. »

Mais, tout ce déroulement ci-dessus n'est qu'un prétexte. "Prendre son café suppose d'abord de passer sur le corps des autres membres de la famille endormis dans le couloir ». Il ne s'agit pas de sagesse dans la vie commune, la hiérarchie doit être toujours au-dessus de tout. Une faute qui méprise le système hiérarchique est un grave problème. Le respect est prédominant dans tous les cas.

La donnée suivante représente une société qui n’apprécie guère l'égalité des sexes. Que le père (représente les hommes) se positionne comme un roi à qui chaque membre de la famille doit s'orienter ou se soumettre.

\section{"Ma femme même, ne peut me donner un si intense plaisir. Croyant, je ne peux, par respect de la tradition, la posséder que dans l'obscurité totale. D'elle je ne connais ce que rapports mes mains... Mon bureau, c'est entier qu'il se livre à moi, à la lumière du jour... De lui, je sais tout : ses petits défauts et ses vices, ses points sensibles et ses zones d'ombres » (Kacimi, 1987, p. 9)}

En lettres grasses, le narrateur fait la comparaison de sa femme à de son bureau (animé et inanimé) ce qui paraît assez absurde. Cela suppose que les citoyens d'un pays peuvent s'enfermer dans l'obscurité, sans avoir le droit d'élargir leur pensée. Par souci de tradition, cette dernière le rend aveugle. Ils doivent se contenter de ce qu'il touche (être soumis à un système) et il n'a pas de droit de regarder plus loin (revendiquer ce qui est naturel pour les hommes).

Cependant, son bureau est une petite pièce où il peut s'exprimer, connaître tous les coins à la lumière du jour. Même si le droit des hommes est très limité, celui qui cherche sa petite liberté trouve une extase dans son fort intérieur. C'est une critique d'une société bourrée et enfermée par une certaine idéologie, qui ne s'ouvre pas pour regarder d'autres possibilités.

\subsubsection{Les symboles dans la technique de conter}

Il n'y a que les personnages ayant un prénom qui sont présentés dans l'histoire : Abdou, Mahfoud et Montalier, les autres ne sont pas présentés. Le narrateur (Monsieur tout le monde), le commissaire, la secrétaire, la femme, l'imam, les responsables sont anonymes.

Il est évident que cet anonymat contribue à l'impression que ce n'est pas réel (ils sont des fantômes, Ils font peur mais ils n'existent pas. En revanche, Abdou, Mahfoud, et Montalier représentent un peuple ayant un comportement distinct.

Abdou : un fonctionnaire de bas niveau qui pose toujours des problèmes au narrateur. Celui qui répand des ondes nauséabondes partout qui expriment son dégoût. (Une dictature du peuple, comportement insolent). Il est aussi raconté que Abdou méprise la peinture de la Joconde (symbole de l'Occident, elle n'est pas voilée) en jaillissant sa morve.

Mahfoud : Celui qui accompagne le narrateur, Il s'éloigne de la vie politique mais aussi en être au courant. Il représente une société qui a peur de lever sa voix pour dire la vérité, que celle-ci est trop passive pour empêcher le mauvais chemin que prend sa société.

Montalier : un français qui est naturalisé algérien et qui vient du parti gauche communiste, détesté par les socialistes. Il est raconté qu'il vient de mourir (assassiné) mais on ne peut pas l'enterrer parce qu'il n'est pas musulman. Puis la solution c'est de le circoncire. (la représentation d'une société dictatoriale)

\subsubsection{La Masturbation est quelque chose de coupable}

"Mon cauchemar, les deux mouchoirs qui attendent au frais que la vérité se fasse jours, le ricanement des employés qui après avoir surpris mon regard porté uniquement sur leurs souliers » (Kacimi, 1987, p. 37)

"Pauvre naïf, voulant à tout prix sauver l'honneur du Parti, j'avais oublié de préserver le mien » (Kacimi, 1987, p. 55) 
Comme le narrateur ne trouve pas celui qui se masturbait ou ceux qui font des rapports sexuels, à la fin il brûle son évidence, ayant peur que cela se retourne contre lui. Comme il s'attachait trop au mouchoir jusqu'à ce qu'il ne fait pas attention à son alentour. Cela donne une preuve de son indifférence. Il représente les hommes, surtout les bureaucrates qui ne parlent pas de ce qu'ils témoignent s'ils trouvaient des faits coupables ou des défauts dans un système. Par contre, la deuxième donnée signifie que dans un grand système véreux, il est dangereux pour ceux qui veulent d'être convenables.

\subsubsection{Un bâtiment de communication}

"Quand il m'arrive de croiser en ville de l'un de ces coupables, je ne le regarde que par transparence. Pour moi il n'est plus individu, mais une longue liste chronologique d'actes répréhensibles. » (Kacimi, 1987, p. 53)

Il est douteux lorsque ce roman ne raconte pas beaucoup d'histoire politique sur le Parti ou ses concurrents dans le bureau. Même l'histoire où le narrateur ou le Parti rendent service aux citoyens.

Même si ce bâtiment était un bureau du Parti Socialiste, il est évident que c'est aussi un bâtiment de communication. C'est à dire un bâtiment qui surveille toutes les activités publiques de ses concitoyens, il n'a jamais fait d'affaire politique ou de service public. Le narrateur surveille et sait facilement tout sur ses concitoyens depuis les archives qu'il a le droit d'accéder. Cela se voit dans la phrase « Pour moi il n'est plus individu, mais une longue liste chronologique d'actes répréhensibles ". Cela est également un signe d'espionnage dans un État totalitaire. Le narrateur connaît toute la vie privée de ses concitoyens, ils n'ont plus d'endroit privé. C'est un pays dictatorial qui surveille ses citoyens afin de préserver le système des gens ayant des idées dangereuses pour lui.

\subsubsection{Un Mouchoir ce n'est pas un mouchoir}

Le Mouchoir est le titre du roman, le narrateur parle quelques fois du mouchoir. Dans l'histoire ce mouchoir s'agit d'une preuve pour garder l'exploit de sperme qu'il a trouvé aux toilettes. Cette preuve a vocation de justifier les faits sexuels qui se sont passés dans les toilettes du deuxième étage par des personnels dans son bureau. C'est toujours une tentative de le conserver, de garder son humidité en le posant devant le climatiseur et la crainte qu'on le trouve en premier et que cela se retourne contre lui. Ce titre ressemble à un mot qui décrit des symboles présentés comme : un bâtiment de communication (espionnage) d'un État totalitaire, c'est un « mouchard » selon Larousse celui-ci a le sens de «personne qui répond les faits et gestes de quelqu'un, qui le dénonce » (Larousse, s. d.). Ce symbole, comme " mouchoir », d'après Riffaterre (1978, p. 2), fait partie de l'ambiguïté de ce sens. Ce mot peut être interprété selon le contexte. Un mouchoir est une chose pour se moucher, nettoyer, et récupérer les choses. Ce mot ressemble beaucoup à un mot « mouchard ». Alors, le mot « mouchoir » veut également dire un mouchard ou un espion ou le fait de faire de l'espionnage

\section{Conclusion}

Le lieu, le temps, et le nom du pays ne sont pas mentionnés dans le roman, cela est peut-être dû pour éviter de porter un jugement direct sur son propre pays. Mais il y a les phrases qui donnent une idée que l'histoire se passe en Algérie, comme décrit au-dessus, le narrateur part pour Oran, la deuxième grande ville d'Algérie. Et il y a des discours sur les militants et anciens combattants qui donnent l'impression que cela se passe juste après la guerre d'indépendance.

La vie du narrateur et ses sentiments en regardant les faits autour de lui comme la coutume dans la famille, la culture de la société et sa vie professionnelle qui représentent sa critique sur la tradition algérienne et la décision ridicule du pays à son regard au sein de la société. Les caractéristiques des personnages sont aussi les symboles du comportement des citoyens du pays dont chacun représente la division d'un comportement générale des algériens. Ces derniers peuvent être trouvés en analysant le Discours dans le roman que l'auteur veut évoquer et le reliant à la vie de l'auteur (Fairclough, 2001, p. 31).

Ensuite, en racontant l'histoire, l'auteur donne des symboles qui ne sont pas assez évidents. On peut être manipulé par l'histoire de ce mouchoir si on ne le lit pas attentivement. Le résultat donne d'autres impressions, observées à partir d'autres points de vue. Mais la critique caricaturale envers son propre 
pays et le titre Le Mouchoir contient une signification d'un comportement d'indifférence du narrateur qui est peut-être mentionné pour renforcer les intellectuels algériens à l'étranger en but d'améliorer sa patrie. Pour finir, le titre Le Mouchoir est le symbole du mot mouchard, qui représente un état totalitaire, le surveillant de ses citoyens jusqu'à leur vie privée, ce qui signifie que la liberté se fane pour les citoyens

\section{Références}

Blanchard, E., \& Thénault, S. (2011). Quel «monde du contact» ? Pour une histoire sociale de l'Algérie pendant la période coloniale. Le Mouvement Social, 236(3), 3-7. https://doi.org/10.3917/lms.236.0003

CRIS (Centre de Ressources Internationales de la Scène). (s.d.). Mohamed Kacimi, actualités, textes, spectacles, vidéos, tous ses liens avec la scène. Retrieved from https://www.theatrecontemporain.net/biographies/Mohamed-Kacimi/presentation/

Fairclough, N. (1995). Critical Discourse Analysis: The Critical Study of Language. London: Longman. https://doi.org/10.2307/329335

Fairclough, N. (2001). Language and Power. New York: Pearson Education Limited.

Kacimi, M. (1987). Le Mouchoir. Paris : L'Harmattan.

Kaltbrunner, D. (1871). Recherches sur l'origine des Kabyles. Le Globe. Revue Genevoise de Géographie, 31-75. https://doi.org/doi.org/10.3406/globe.1871.4295

Larousse. (s.d.). Mouchard. Retrieved from https://www.larousse.fr/dictionnaires/francais/mouchard_moucharde/52847

Riffaterre, M. (2006). Semiotics of Poetry. Bloomington: Indiana University Press. https://doi.org/10.2307/429927

Zenati, J. (2004). L'Algérie à l'épreuve de ses langues et de ses identités : histoire d'un échec répété. Mots. Les langages du politique, 74(2), 137 145. Retrieved from https://www.cairn.info/load_pdf.php?ID_ARTICLE=MOTS_074_0010 